\title{
The Gradient of $\gamma$-Rays Irradiation's Energy and Its Use in Treatment of Cancer Diseases
}

\author{
Labinot Kastrati' ${ }^{1}$, Gazmend Nafezi ${ }^{2}$, Gëzim Shehi ${ }^{3}$ \\ ${ }^{1}$ Department of Radiotherapy, Clinic of Oncology, University Clinical Center of Kosovo, Pristina, Kosovo \\ ${ }^{2}$ Faculty of Mathematical and Natural Sciences, University of Pristina, Pristina, Kosovo \\ ${ }^{3}$ Department of Physics, Faculty of Natural Sciences, University of Tirana, Tirana, Albania \\ Email: "gazmend_nafezi@hotmail.com
}

Received 8 March 2016; accepted 23 May 2016; published 26 May 2016

Copyright (C) 2016 by authors and Scientific Research Publishing Inc.

This work is licensed under the Creative Commons Attribution International License (CC BY).

http://creativecommons.org/licenses/by/4.0/

CC) (i) Open Access

\section{Abstract}

The Ionising irradiations used mostly in the treatment of tumoral diseasses are: $\mathbf{X}, \gamma, \boldsymbol{\beta}$ and $\mathbf{e}$ irradiations. The discussion will be about $\gamma$ irradiations, produced in linear accelerator with photon energy $6 \mathrm{MV}$ and $15 \mathrm{MV}$. It is important to know the absorption performance before and after the electronic equilibrium. This is a reason that we've used the function of dose gradient for irradiations $\gamma$. It represents the velocity of dose change as a function of depth in tissue. From skin to maximum dose value, the increase of G-function is more accentuated for $\gamma$-rays than for $\beta$-particles while after that the G-function decreasing is less sharp for $\gamma$-rays. Finally, we'll discuss about the advantages in terms of radiation protection of $\gamma$-rays used in radiotherapy.

\section{Keywords}

$\gamma$-Rays, G-Function, Photon Energy, Radiation Protection

\section{Introduction}

When an irradiationbeam passes from a point $A$ to an other point $B$, the energy of beam changes from $E_{1}$ to $E_{2}$. The difference $\Delta E=E_{1}-E_{2}$ is the lost energy by the interaction of beam itself with material of tissue. Gradient of beam's energy would be:

$$
\frac{\Delta E}{d}=\frac{\left(E_{1}-E_{2}\right)}{D}=G
$$

As we can see, this mathematical function means the rate or the velocity of beam's energy reduction (de-

\footnotetext{
${ }^{*}$ Corresponding author.
}

How to cite this paper: Kastrati, L., Nafezi, G. and Shehi, G. (2016) The Gradient of $\gamma$-Rays Irradiation's Energy and Its Use in Treatment of Cancer Diseases. International Journal of Medical Physics, Clinical Engineering and Radiation Oncology, 5, 150-153. http://dx.doi.org/10.4236/ijmpcero.2016.52016 
creasing). This dosimetric quantity expressed in math's terms is investigated in this work to increase the beam's irradiation efficiency, as much as possible to the tumoral cells, protecting at the same time the healthy tissues and organs near and around [1]-[4]. To do this, we used in radiotherapy $\gamma$-rays of different energies. The loss of energy for $\gamma$-rays, mostly is realised through indirect ionisation, because the direct one throughout the direct collision is almost negligible, is almost zero, to the fact that $\gamma$-rays are electromagnetic waves without electric charge, without mass, and of course there is no sense, no meaning to discuss about the dimensions of $\gamma$-rays. Consequently, the probability of direct collisions with atomic structures of absorbing background is closely to zero, while its dependence by the nominal energy is very small, too. In Figure 1, we have illustrated the weak dependence of radiation performance of X-rays during their spread in tissues. In the same figure, there shows three cases of isodose curves spreadout for $\gamma$-rays of different energies. Compton effect and pair creation effect about which we have to disscuss more detailly below, are the "mecanism" through which $\gamma$-rays cause ionisation.

The main characteristics of G-function for $\gamma$-rays are: on the first impact in skin surface, the production of the ions is intensive, to the drastic change of air to tissue density. Nevertheless, almost the half of ions go back or scattered back, while the parts which continue their paths inside, continue the ionisation. But now, together with the negative part of ions, the electrons can already cause a supplementary, more intensive ionisation, like electrons. Electrons created in the first millimeters of tissue, increase the number of electron's interactions, while the decrease is by exponencial law with a normal impedance, which depends from the energy of $\gamma$-rays, as well as by the tissues density. G-function would be an increasing function up to a depth, which depends by the $\gamma$ photon's energy, where the electrons lose their energy and G-function begins to decrease. The zone where G-function changes the sign, from increasing to decreasing function, or mathematically from positive values to negative values, is the zone where the number of created secondary electrons is equal with the number of absorbed one, for any unit of time. Do to the relative low intensity of $\gamma$-rays interactions behind this zone, and do to fast absorbtion of secondary electrons, $\gamma$-rays continue its path in health structures, giving unnecessary irradiation to there [5].

\section{Material and Methods}

Material is took of from the every day typical treatment cases,using the real data of patients. In Figure 2, it is shown a picture of Percentage Depth Dose (PDD) for $(10 \times 10)$ beam size of $\gamma$-rays, generated in accelerator with energy of $6 \mathrm{MV}$.

The generating energies, are less or more of nearlythe same valueswithin the possibilities available in our institution. This, I think is necessary to do the comparison more credible. As we can see, the first part of curve, that from the skin to the maximal value(which is took as $100 \%$ ), increase a litle bit fastly.

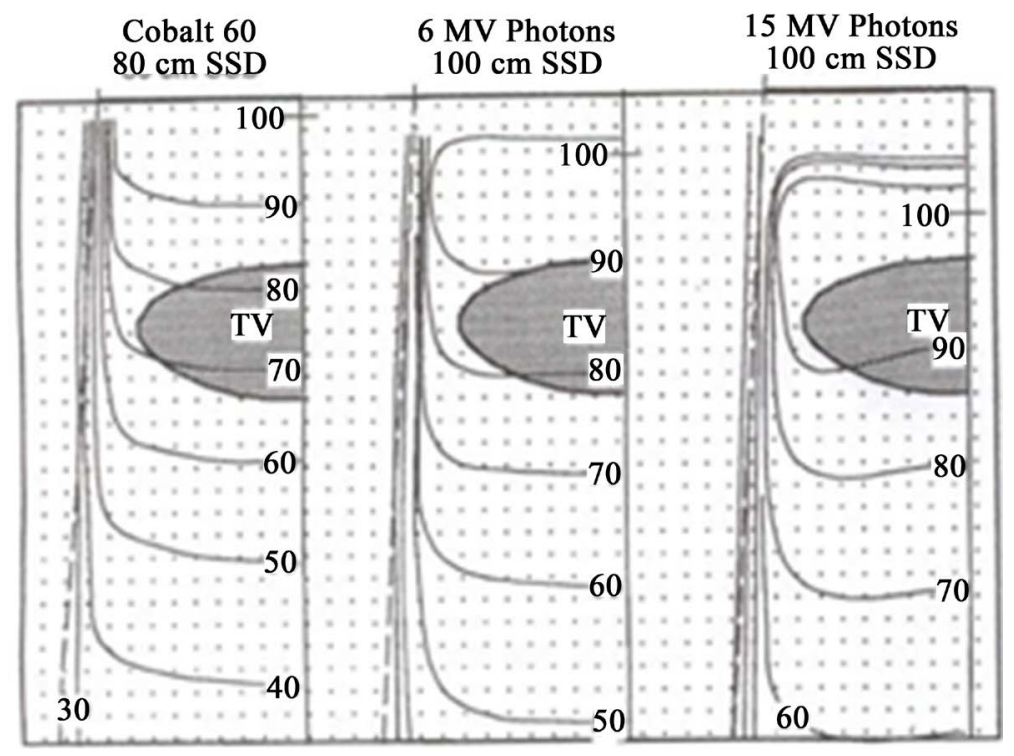

Figure 1. Three cases of isodose curves spreadout for $\gamma$-rays of different energies. 


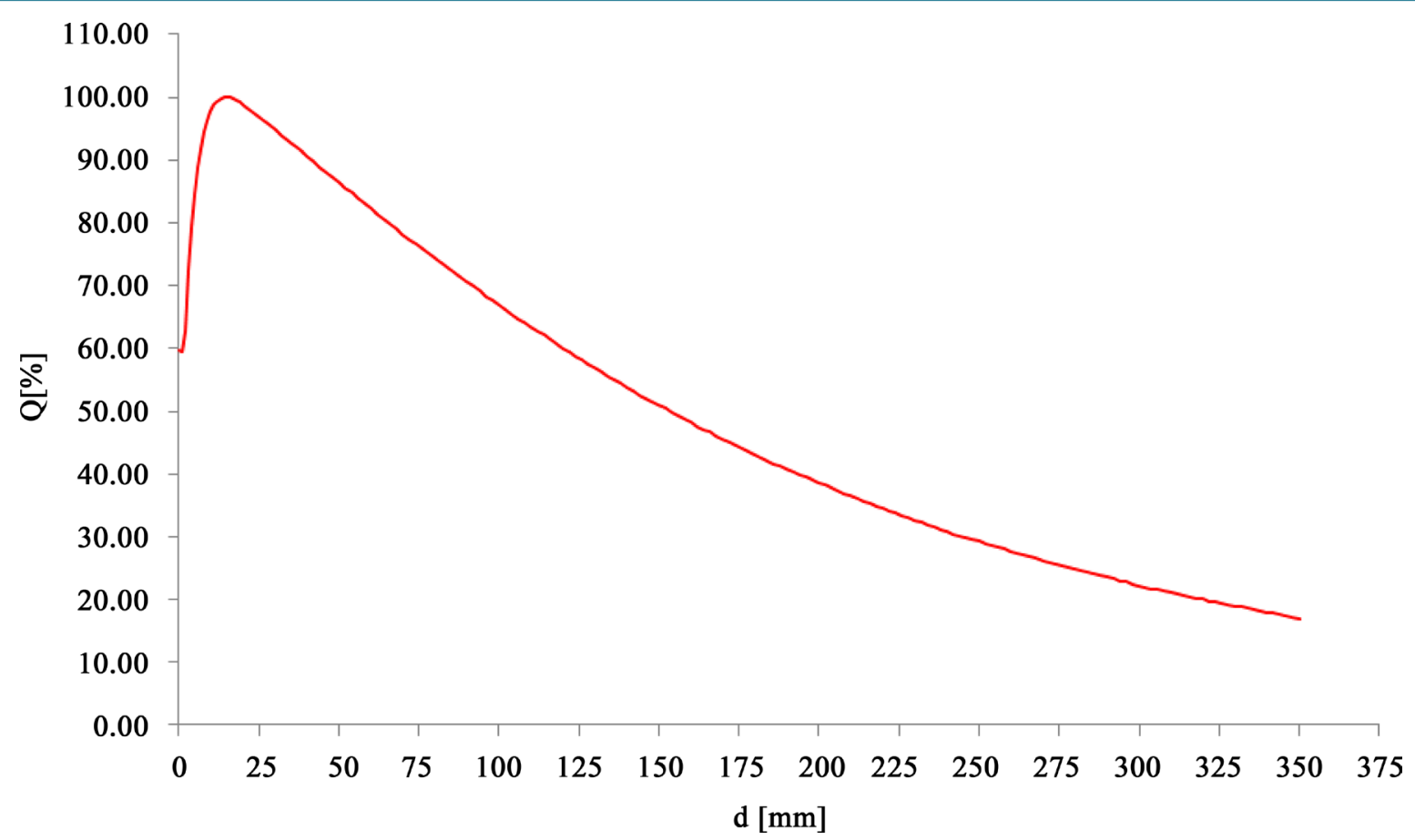

Figure 2. PDD for photons with energy $6 \mathrm{MV}$, field size $10 \mathrm{~cm} \times 10 \mathrm{~cm}$.

The total G-function determined from maximal values to value zerowill be ecxtremely higher, because the value zero in this case is not reached or as is accept to speak, is reached in infinit.Qualitatively, these two values can't be paragonate and for these reasons, in generally are paragonate the values of $80 \%$ or/and $50 \%$. The second value, i.e. $50 \%$ will be: $50 \% / 160 \mathrm{~mm}=3.125 \% / \mathrm{cm}$. As we can see from Figure 2 , this rate is not constant, it is an increasing function mostly, because this part of graf. In Figure 2, decrease asymptotically to the $\mathrm{X}$-axis, which means the function itself never touch it or the dose value never arrive the value zero [6].

\section{Discussion}

The depth, which in the case of cobalt- 60 photons is $5 \mathrm{~mm}$, in the similar energy photons produced in accelerator is $16 \mathrm{~mm}$. For the PDD distribution dependence of isodose curves from energy, we can see in Figure 1 , that if the maximal dose curve, that of Co-60 photons, is an open curve at $5 \mathrm{~mm}$ depth (a), for $6 \mathrm{MV}$ energy photons of accelerator is not only in a greater depth, as we underline above, this is more important, but the curve of maximal dose is already a closed curve (b). If the energy is higher (case (c), in the same Figure, the curve of $100 \%$, is not only closed, but it is in a greater depth, which is about $20 \mathrm{~mm}$. Being closed curves, they includes a surface starting from: 16 to $42 \mathrm{~mm}$, in (b) case, to about $20 \mathrm{~mm}$ to $56 \mathrm{~mm}$ in (c) case; from which we can see that the closed zone increase the deep of its location with increasing of energy, as well surface of zone with maximal energy. This kind of distribution is charachteristic for high energy $\gamma$-rays, generated in accelerators.

The most important discussion's argument to keep in mind is:to do maximal caution, necessary to realise the quality assurance(QA) treatment, to perform a correct treatment, being this one always more than necessary. This becomes more important, even imperative, because the treatment is based in high energy radiations, which, if they are used without necessary precaution and attention, can becomes not efficient, or even dangerous for the patient. To avoid such kind of accidents, it is more than necessary to do a good simulation, and after this to do the controls for any patient, simulating in detailsevery step, before the treatment itself. All parameters must be controlled, using an highly exact quality controll, an exact simulation of individual characteristics of each patient.

\section{Conclusion}

From the skin to the maximal value of PDD for the same beam size, energy etc., the G-function of irradiation is almost the same. The G-function, after the maximal dose value, is a descending function for $\gamma$-rays. This enables 
almost perfectly the protection of behind tumor structures.

\section{Acknowledgements}

I gratefully acknowledge the staff of Institute of Oncology in Kosovo, especially the staff of physicists in Department of Radiotherapy.

\section{References}

[1] Bentel, G.C. (1996) Radiation Therapy Planning. McGraw-Hill, New York.

[2] Khan, F.M. (2010) The Physics of Radiation Therapy. Lippincott, Williams and Wilkins, Baltimore.

[3] Beyzadeoglu, M., et al. (2010) Basic Radiation Oncology. Springer, New York.

[4] Cember, H. (2009) Introduction to Health Physics. McGraw-Hill, New York.

[5] (2004) National Program for Radiotherapy in 2010-2013 Period in Albania.

[6] Xhafa, B., et al. (2014) Dose Distribution of Photon Beam by Siemens Linear Accelerator. International Journal of Medical Physics, Clinical Engineering and Radiation Oncology, 3, 67-70. http://www.scirp.org/journal/ijmpcero 